\title{
CORRESPONDENCE
}

\section{Are PCR Artifacts in Microdissected Samples Preventable?}

To the Editor:-In many instances, molecular results are determined by technical conditions and need validation. Loss of heterozygosity (LOH) and microsatellite instability (MSI) analyses share these limitations too, but only a few articles directly study the technical aspects of molecular tests.

Sieben et al address technical issues of $\mathrm{LOH}$ and MSI analysis using small amounts of DNA (microdissected tumor samples). ${ }^{1}$ The study design is appropriate for the objectives, but the technique implications are quite extensive and a broader discussion on biological and technical aspects would help both interpreting and applying those analyses. The authors clearly show the inverse relationship between the concentration of the target DNA and the presence of test artifacts, but can those artifacts be explained by the DNA concentration only? If that is the case, are the molecular results based on single-cell analysis believable? Some thoughts trying to answer these questions are provided.

The identification of extrabands in the amplification product from a polymorphic DNA region will be considered evidence of MSI if they are not present in the corresponding control. $^{2-5}$ To avoid misinterpretations, the amount of tissue in control and test samples should be similar, thus correcting biased patterns caused by artifacts induced by small target concentrations in tumor samples only. ${ }^{5}$ Those additional bands must be carefully distinguished from polymerase chain reaction (PCR) artifacts and different options must be investigated and systematically excluded:

1. Target DNA modifications induced by fixation and processing in paraffin-embedded tissues. The fixation process involves multiple cross-links between amino-groups of nucleic acid bases and polypeptide aminoacids to preserve tissue morphology. ${ }^{6}$ A side effect of the tissue processing is a variable nucleic acid fragmentation and denaturation that result in the typical smear pattern of DNA extracted from paraffin-embedded tissues. The covalent links between different DNA fragments will determine the presence of single-stranded sequences with $3^{\prime}-\mathrm{OH}$ free that can act as primers during the polymerization step of PCR, the so-called primer-independent DNA amplification. ${ }^{6}$ A prolonged protein digestion in the appropriate conditions during the DNA extraction significantly reduces the number of crosslinks, thus resulting in better definition of the amplification product.

2. The repetitive sequence would result in a higher incidence of primer misannealing and hairpin formation. These possibilities can be avoided setting a long denaturation in the first few cycles when the template DNA is mainly genomic and including 7-deaza-2'-dGTP in the reaction mixture if the target is a CG-rich sequence. This nucleotide substitution during amplification reduces stability of intramolecular and intermolecular GC base pairing and avoids biased target amplification. ${ }^{7}$ These 2 technical modifications favor proper annealing and amplification of the specific target. ${ }^{7-9}$

3. The amplification conditions can also determine the specificity of the reaction. Both nucleotide concentration and the labeling methods have been reported limiting factors. ${ }^{2,5}$ The probability of getting additional bands decreases when nucleotide concentration is reduced and an external labeling method (only one primer labeled) is used.

The presence of minute DNA amount in the reaction mixture results in more frequent PCR artifacts, especially if the target DNA is fragmented. In microsatellite analysis, Sieben et al report a significant increase of these PCR artifacts when the amount of target DNA is lower than $5 \mathrm{ng}$ (DNA isolated from frozen tissues) or $10 \mathrm{ng}$ (DNA isolated from formalin-fixed, paraffin-embedded tissues).${ }^{1}$ Considering the DNA amount per cell $(\sim 7 \mathrm{pg})$, the limiting DNA amount would represent between $\sim 700$ cell equivalents (frozen tissues) and $\sim 1,400$ cell equivalents (formalin-fixed, paraffinembedded tissues). This issue is extremely important for microdissected samples because they normally contain less DNA. However, the relative incidence of these artifacts can be controlled by applying the previously described principles, which bypass the most frequent causes of these artifacts. This also emphasizes the importance of careful method design to obtain reliable results in molecular analyses.

The high incidence of PCR artifacts using microdissected samples is related to the small concentration of target DNA, fixation-induced changes of DNA, and conditions in the amplification of repetitive sequence (especially for those CG-rich sequences) favoring misannealing and hairpin formation. Appropriate modifications to avoid the previously mentioned conditions will significantly improve the reproducibility of $\mathrm{LOH}$ and MSI test in microdissected samples.

SAlvador J. Diaz-Cano, MD, PHD
Department of Histopathology
Bart's and The London Queen Mary's
School of Medicine and Dentistry
University of London
London, England

1. Sieben NL, ter Haar NT, Cornelisse CJ, et al: PCR artifacts in LOH and MSI analysis of microdissected tumor cells. HuM PATHOL 31:1414-1419, 2000

2. Koreth J, O'Leary JJ, McGee JOD: Microsatellites and PCR genomic analysis. J Pathol 178:239-248, 1996

3. Diaz-Cano SJ, Blanes A, Rubio J, et al: Molecular evolution and intratumor heterogeneity by topographic compartments in muscle-invasive transitional cell carcinoma of the urinary bladder. Lab Invest 80:279-289, 2000

4. Diaz-Cano SJ: Designing a molecular analysis of clonality in tumours. J Pathol 191:343-344, 2000

5. Diaz-Cano SJ, Blanes A, Wolfe HJ: PCR techniques for clonality assays. Diagn Mol Pathol 10:24-33, 2001

6. Diaz-Cano SJ, Brady SP: DNA extraction from formalin-fixed, paraffinembedded tissues: Protein digestion as a limiting step for retrieval of highquality DNA. Diagn Mol Pathol 6:342-346, 1997

7. Mutter GL, Boynton KA: PCR bias in amplification of androgen receptor alleles, a trinucleotide repeat marker used in clonality studies. Nucleic Acids Res 23:1411-1418, 1995

8. Diaz-Cano SJ, de Miguel M, Blanes A, et al: Clonality as expression of distinctive cell kinetics patterns in nodular hyperplasias and adenomas of the adrenal cortex. Am J Pathol 156:311-319, 2000

9. Diaz-Cano SJ, de Miguel M, Blanes A, et al: Clonal patterns in phaechromocytomas and MEN-2A adrenal medullary hyperplasias: Histologic and kinetic correlates. J Pathol 192:221-228, 2000

doi:10.1053/hupa.2001.29632 
Reply

To the Editor:-We would like to thank Dr Diaz-Cano for his comments on our recent article describing polymerase chain reaction (PCR) artifacts in loss of heterozygosity ( $\mathrm{LOH})$ and microsatellite instability (MSI) analysis of microdissected tumor cells. It is becoming increasingly apparent that significant problems may be encountered during attempts to unambiguously reproduce LOH and MSI data based on DNA obtained from microdissected tumor tissue. Although more than one factor may play a role, the concentrations of template DNA used are seldom accurately quantitated and may vary greatly between the individual tumor samples and corresponding normal tissue. To identify the necessary parameters for facilitating reliable PCR-based analyses, we performed PCR with increasing dilutions of template DNA in nearly 1,000 PCR reactions. By doing so, we showed a significant association between the concentration input DNA used and the percentage of artifactual LOH or MSI observed. Based on this study, a minimum of $5.0 \mathrm{ng}$ for fresh frozen tissue and $10.0 \mathrm{ng}$ for formalin-fixed, paraffin-embedded tissue is set for reliable PCR analysis under our experimental conditions.

We agree with Dr Diaz-Cano that other factors such as tissue-fixation method and processing influences DNA quality and thus optimal PCR amplification. For example, tissue fixation in ethanol is less deleterious for DNA than formalin and less input DNA is necessary to obtain reproducible results. We can fully subscribe the importance of optimal test conditions in microsatellite analysis, one of which should be DNA quantification and input standardization, and we do not seem to deviate from Dr Diaz-Cano's conclusions.

Nathalie L. Sieben, MD Anne-Marie Cleton-Jansen, PhD Department of Pathology Leiden University Medical Center Leiden, The Netherlands

doi:10.1053/hupa.2001.29633

\section{Tumor Invasion and Metastasis-Nature or Nurture?}

To the Editor:-In the article by Matias-Guiu, ${ }^{1}$ it is stated that "Once the tumor has developed, several additional molecular abnormalities occur in different neoplastic subclones; these new alterations are responsible for tumor heterogeneity, tumor invasion, and metastasis." In the accompanying editorial, ${ }^{2}$ it is stated that "Tumorigenesis is conceived as involving a stepwise accumulation of genetic damage within a cell that ultimately undergoes malignant transformation and, in time, the proliferation of clones having such phenotypic properties as drug- or hormone-resistance, invasion, or metastasis." Also in the editorial it is stated that "Additional molecular changes occurring in different neoplastic subclones are responsible for the clinically apparent phenotypes of tumor invasion and metastasis."

Strongly implied but without proof is that some genetic alteration(s) transforms a dividing cell into a cell that can detach itself from its neighboring tumor cells, travel from one location to another, and enter into veins and/or lymphatics (invasion). Further, the genetic alteration(s) of the dividing cell transforms the cell into one that can now find nourishment, continue to divide, and avoid being destroyed by the body's immune defenses (metastasis) at some distant location.
There is nothing in the article by Matias-Guiu, nor in the editorial, that would support any theories other than that genetic mutations occur and occur sequentially with time. The functional change brought about by any given alteration in DNA is speculative at best, when considering invasion and metastasis. That such genetic events lead to a change in the cell's ability to divide or not to divide seems to be an acceptable conclusion from what is known of cell division mechanisms. However, to conclude that genetic events are responsible for invasion and metastasis is premature. ${ }^{3}$

Elsewhere, ${ }^{3}$ I have reintroduced an old concept that will account for invasion and distant transport of tumors invoking only the normal physiologic and mechanical processes of traumatic separation of tumor cells from anchoring neighbors, traumatic disruption of basement membrane material, lymphatic flow dynamics, and the anatomic introduction of lymph-containing tumor cells and debris into the venous system to widely disseminate tumor cells throughout the body. The mechanism of establishing a stable and/or growing metastasis may as likely reside in the host defenses as in the tumor cell's DNA.

Increasing numbers of genetic alterations may be important to increasing cell division and decreasing cell destruction (apoptosis), but there is no inherent biologic necessity to invoke genetic events into the invasion and metastasis process.

\section{ROBERT J. ROSSER, MD Pathology Department Desert Regional Medical Center Palm Springs, $C A$}

1. Matias-Guiu X, Catasus L, Bussaglia E, et al: Molecular pathology of endometrial hyperplasia and carcinoma. Hum Pathol 32:569-577, 2001

2. Foster CS, Gorstein F: Molecular basis of disease processes. Hum PATHOL 32:567-568, 2001 (editorial)

3. Rosser RJ: A point of view: Trauma is the cause of occult micrometastatic breast cancer in sentinel axillary lymph nodes. The Breast Journal 6:209212, 2000

doi:10.1053/hupa.2001.29636

\section{Reply}

To the Editor:-We would like to express our appreciation to Dr Robert J Rosser for his interest in our review article, "Molecular Pathology of Endometrial Hyperplasia and Carcinoma." There is a large body of evidence in the medical literature suggesting that some genetic alterations are associated with the development and progression of human carcinomas. Our article summarizes the results obtained by several authors, including ourselves, in understanding the role of certain genetic abnormalities in the appropriate morphologic context. Likewise, it is understood that the process of tumor progression includes many other aspects such as host response and functional disruption of the relation between epithelial cells and their environment.
Xavier Matias-Guiu, MD
Jaime Prat, MD, FRCPath
Department of Pathology
Hospital de la Santa Creu i Sant Pau
Barcelona, Spain

doi:10.1053/hupa.2001.29637 


\section{Editors' Reply}

The editorial that accompanied the article by MatiusGuiu et $\mathrm{al}^{1}$ in the June issue of Human Pathology was written to challenge several currently held concepts and to highlight a series of issues presently being raised by a wide range of different molecular biological studies. Intentionally, the editorial was not restricted to a consideration of only those concepts that were raised in the article concerning the molecular pathology of endometrial hyperplasia and carcinoma. It is both unfortunate and unintentional if there was any apparent ambiguity in the content of the editorial such that it seemed to imply that specific genetic alterations are required to transform a cell into one that can become metastatic, as indicated in the accompanying letter by Dr Rosser. If there has been such an implication or interpretation, then we apologize that the sense of the editorial was not conveyed with greater clarity.

Rather than being at variance, we agree with Dr Rosser that there is no evidence for the existence of specific metastasis genes or for specific genetic alterations that might be construed as metastasis genes. Although current evidence points to the initiation of neoplasia involving specific genetic events, the same is not true of the metastatic process. One difficulty now facing cancer researchers is the separation of those molecular and cellular events that are causal to the metastatic process from the myriad of epiphenomena that occur during the evolution and progression of each malignancy. Thus, we concur with Dr Rosser that neither the article by Matius-Guiu et al nor our editorial supports any theories other than the temporal occurrence of genetic mutations with tumor advancement.

In his letter, Dr Rosser raises an important problem with respect to the biology of the metastatic process that may be addressed, in part, by consideration of events that occur in nonmalignant tissues. During embryogenesis and tissue morphogenesis, the phenotypic properties of cellular diversion, migration, and invasion are both common and are characteristic attributes of benign cells. Even during postembryonic maturation, some cell types (eg, interdigitating dendritic cells within the skin ${ }^{2}$ and normal B-lymphocyte subsets within the ileum and salivary glands ${ }^{3}$ ) undergo migration, differentiation, homing, and tissue invasion. These observations related to mesenchymal cells within the reticuloendothelial system might be considered to be intrinsically migratory. However, recent independent observations have shown that cells of adult hemopoietic origin can invade the human liver with the result that they repopulate the epithelial compartment, initially as hepatocytes. ${ }^{4}$ Further, migration and differentiation of these cells also result in the generation of cholangiocytes. ${ }^{5}$ With respect to epithelial malignancy, additional evidence is accumulating that several proteins essential to the metastatic process are the normal products of nonmutated genes-but aberrently expressed or regulated by the metastatic cells. Amongst others that are now being described, these include the $\mathrm{Ca}^{++}$-binding protein $\mathrm{p} 9 \mathrm{Ka},{ }^{6}$ voltage-gated $\mathrm{Na}^{+}$channels, ${ }^{7} \mathrm{C}$-FABP, 8 and fascin, ${ }^{9}$ a molecule essential to the migration and differentiation of interdigitating dendritic cells within the skin.

In contrast to oncogenic genes that are mutated and encode abnormal products, evidence currently accumulating suggests that metastasis-promoting genes are nonmutated and yield normal proteins. Furthermore, there is no evidence to indicate that activity of metastasis-promoting proteins are obligated to any of the mutational events that are characteristic of or accompany tumor progression. Elucidation of this relationship is likely to provide key information concerning the biological nature of the metastatic process.

Christopher S. Foster, MD, PhD, FRCPath Editor, European Editorial Office

University of Liverpool

Liverpool, England

Fred GORSTEIN, MD
Editor
Thomas Jefferson University
Philadelphia, PA

1. Matias-Guiu X, Catasus L, Bussaglia E, et al: Molecular pathology of endometrial hyperplasia and carcinoma. Hum PATHOL 32:569-577, 2001

2. Caux C, Ait-Yahia S, Chemin K, et al: Dendritic cell biology and regulation of dendritic cell trafficking by chemokines. Springer Semin Immunopathol 22:345-369, 2000

3. Woodruff JJ, Katz M, Lucas LE, et al: An in vitro model of lymphocyte homing II. Membrane and cytoplasmic events involved in lymphocyte adherence to specialized high-endothelial venules of lymph nodes. J Immunol 119: $1603-1610,1977$

4. Alison MR, Poulsom R, Jeffery R, et al: Hepatocytes from non-hepatic adult stem cells. Nature 406:257, 2000

5. Theise ND, Nimmakayalu M, Gardner R, et al: Liver from bone marrow in humans. Hepatology 32:11-16, 2000

6. Ke Y, Jing C, Barraclough R, et al: Elevated expression of calciumbinding protein $\mathrm{p} 9 \mathrm{Ka}$ is associated with increasing malignant characteristics of rat prostate carcinoma cells. Int J Cancer 71:832-837, 1997

7. Smith P, Rhodes NP, Shortland AP, et al: Sodium channel protein expression enhabces the invasiveness of rat and human prostate cancer cells. FEBS Letters 423:19-24, 1998

8. Jing C, Beesley C, Foster CS, et al: Human cutaneous fatty acid-binding protein induces metastasis by up-regulating expression of vascular endothelial growth factor gene in rat RAMA 37 model cells. Cancer Res 61:4357-4364, 2001

9. Yamashiro S, Yamakita Y, Ono S, et al: Fascin, an actin-bundling protein, induces membrane protrusions and increases cell motility of epithelial cells. Mol Cell Biol 9:993-1006, 1998

doi:10.1053/hupa.2001.29638 